\title{
Coerción sexual, compromiso y violencia en las relaciones de pareja de los universitarios
}

\author{
Elena Hernández González y Rosaura González Méndez
}

\author{
Universidad de La Laguna
}

Disponible online 30 de agosto de 2009

\begin{abstract}
Se analizan las tácticas de coerción sexual en parejas de universitarios, y su asociación con el tipo de compromiso con la relación y la violencia física y sexual sufridas. Participaron 175 estudiantes, 79 mujeres y 96 hombres. A partir de la propuesta de DeGue y DiLillo (2005), se desarrolló una nueva escala que mide coerción sexual. Los análisis factoriales exploratorios revelaron tres componentes: Insistencia, Chantaje Emocional y Culpabilización. La agresión sexual se midió con un único ítem. El compromiso y la violencia física sufrida se midieron mediante escalas ya existentes. El análisis discriminante confirmó que el grupo que sufre violencia física (victimizado) difiere significativamente del que no la sufre (no victimizado). Las variables que contribuyeron a dicha clasificación fueron el Compromiso Negativo y la Insistencia para el primer grupo, y el Compromiso Positivo para el segundo. Se discuten las implicaciones de los resultados para la prevención de la violencia.
\end{abstract}

Palabras clave:

Coerción sexual, violencia de pareja, compromiso

This study analyzes the tactics of sexual coercion among university couples, and their association with the type of commitment with the relationship and experienced physical and sexual violence. Participants were 175 college students, 79 women and 96 men. A new sexual coercion scale was developed, based on the DeGue and DiLillo's (2005) proposal. The exploratory factor analyses showed three components: Insistence, Emotional Manipulation, and Blame. Sexual assault was measured with a single item. Commitment and experienced Physical Violence were measured using existing scales. Discriminant analysis confirmed that participants from the victimized group significantly differed from those of the non-victimized group. The variables that significantly contributed to the classification were Negative Commitment and Insistence for the victimized group and Positive Commitment for the non-victimized group. We discuss the implications of the results to prevent partner violence.

Keywords:

Sexual coercion, partner violence, commitment

Correspondencia: Rosaura González Méndez. Universidad de La Laguna. Facultad de Psicología. Campus de Guajara. 38205 La Laguna, Tenerife. Fax: 9223174 61. Email: mrglez@ull.es 
Las mujeres que sufren violencia dentro de sus relaciones de pareja suelen estar expuestas a más de una forma de agresión (Vézina y Hébert, 2007). Así, la mayor parte de las mujeres que experimentan violencia física son además objeto de maltrato psicológico; y entre un tercio y la mitad de las que son maltratadas físicamente, también son víctimas de agresiones sexuales (Krug, Dahlberg y Mercy, 2002).

De las distintas formas de violencia que ocurren dentro de las relaciones de pareja, la sexual sigue siendo la menos "visible". Así, resulta poco conocido que, para una tercera parte de las adolescentes de todo el mundo, su primera experiencia sexual sea forzada (Krug et al., 2002). Por otro lado, tampoco suele mencionarse que la violencia durante el noviazgo sea un problema propio de culturas donde la actividad sexual prematrimonial está normalizada; mientras que el riesgo de violencia se pospone hasta después de la boda en aquellas otras donde dicha práctica sexual es poco frecuente (Jewkes, 2002). En este sentido, y puesto que la juventud en nuestro país es cada vez más precoz en su iniciación sexual (Serapio, 2006), resulta comprensible la elevada prevalencia que tienen tales agresiones entre los adolescentes y jóvenes españoles (Fernández-Fuertes, Fuertes y Pulido, 2006; González y Santana, 2001; MuñozRivas, Graña, O’Leary y González, 2007).

En los últimos años, la investigación ha aportado mucha información sobre la prevalencia y los factores de riesgo asociados a la violencia que afecta a las parejas jóvenes (v. g. Lewis y Fremouw, 2001), pero apenas se ha investigado cómo se gesta dicha dinámica. No obstante, los datos anteriores sugieren que la actividad sexual puede ser uno de los escenarios donde comienza a manifestarse el problema. De hecho, la violencia sexual puede comenzar antes incluso que la violencia física (Harner, 2002).

Al tratar de delimitar qué se entiende por agresión sexual, encontramos definiciones de diversa amplitud (Muehlenhard y Peterson, 2005). Mientras las más restrictivas abarcan únicamente las formas de agresión más evidentes y extremas; las más amplias incluyen también formas sutiles, que no implican el uso de fuerza física (chantaje, insistencia verbal, amenaza de ruptura...). Para estas últimas, sin embargo, algunos investigadores reservan el término coerción sexual, que arrastra también cierta imprecisión (DeGue y DiLillo, 2005).

Para Adams-Curtis y Forbes (2004), por ejemplo, la coerción sexual es cualquier situación en la que se emplean métodos verbales o físicos para conseguir una actividad sexual, que no es consentida libremente. En la misma línea, se la considera como un abanico de estrategias que van desde el uso de la fuerza, la amenaza de violencia o la intoxicación, hasta fórmulas más sutiles como la manipulación emocional (v.g. Shackelford y Goetez, 2004).

Sin embargo, otras definiciones incluyen exclusivamente tácticas no físicas, siendo el empleo de la fuerza el aspecto diferenciador entre coerción y agresión sexual (DeGue y
DiLillo, 2005; Testa y Dermen, 1999). En esta línea, DeGue y DiLillo (2005) definen la coerción sexual como un tipo de comportamiento sexual inapropiado, que consiste en usar tácticas no físicas (v. g. presión verbal, mentiras y disputas continuas) para conseguir un contacto sexual no deseado. Asimismo, clasifican los comportamientos sexuales mediante el cruce de dos dimensiones: a) tipos de contacto sexual; y b) tácticas utilizadas para obtener dicho contacto. La primera dimensión representa un continuo que va desde las caricias y besos hasta la penetración. La segunda comprende desde la coerción verbal más sutil hasta el uso de la fuerza.

De acuerdo con este modelo, asumimos que la coerción sexual es una forma de conducta sexual abusiva, que se manifiesta a través de tácticas no físicas, y que es independiente de los actos sexuales que deriven de ellas. Asimismo, entendemos que la agresión sexual implica el uso de fuerza física, también con independencia de la actividad sexual resultante.

La mayor parte de los instrumentos que miden coerción sexual han sido diseñados para analizar los encuentros ocasionales, y no las relaciones de pareja. La investigación sugiere, sin embargo, que las estrategias utilizadas en ambos casos pueden ser diferentes (Shackelford y Goetz, 2004; Testa, VanZile-Tamsen, Livingston, 2007).

Por otro lado, los instrumentos desarrollados para medir la coerción sexual en las parejas también presentan algunas limitaciones (Shackelford y Goetz, 2004). Por ejemplo, la escala más utilizada hasta el momento, la Sexual Experience Survey (SES; Koss y Oros, 1982), valora la coerción a lo largo de vida, y no permite conocer el momento, la frecuencia y el número de parejas con las que ha ocurrido. Otra medida muy utilizada, la sub-escala de coerción sexual de la Revised Conflict Tactics Scales (CTS2; Straus, Hamby, Boney-MacCoy y Sugarman, 1996), mide coerción en general y no distingue entre diferentes estrategias.

Uno de los aspectos que ha orientado la investigación sobre coerción sexual ha sido, precisamente, delimitar las diferentes tácticas utilizadas. Sin embargo, no suele diferenciarse entre coerción y agresión. Por ejemplo, la Sexual Coercion in Intimate Relationships Scale (SCIRS; Shackelford y Goetz, 2004) identifica tres tipos de estrategias. No obstante, mientras dos de ellas son coercitivas, manipulación del compromiso (se esgrime que el afecto y el compromiso conllevan obligación) y amenaza de desafección (se induce miedo al abandono), la tercera, manipulación de recursos/violencia, mezcla indicadores de coerción y agresión.

En la misma línea, Livingston, Buddie, Testa y VanZileTamsen (2004) identificaron cuatro tipos de estrategias: Persuasión Verbal, Insistencia, Persuasión Física y Ganancia de Acceso. Dentro de la Persuasión Verbal, distinguen entre tácticas negativas, positivas y neutras. Las negativas incluyen expresiones de descontento, agresiones verbales, retraimiento, 
etc. que suscitan culpa o miedo al abandono. Las positivas incluyen la seducción con palabras; y las neutras no tienen carga emocional. La Insistencia incluye tácticas verbales o físicas usadas persistentemente. La Persuasión Física comprende desde besos y caricias hasta agresiones sexuales. Por último, la Ganancia de Acceso supone buscar excusas para aproximarse.

Finalmente, Struckman-Johnson, Strucman-Johnson y Anderson (2003) separan coerción y agresión sexual en diferentes niveles de abuso, y proponen cuatro tipos de tácticas, que suponen niveles crecientes de gravedad: nivel 1, tácticas no verbales de excitación sexual (persistir con caricias, quitar la ropa...); nivel 2, tácticas de manipulación emocional (insistencia verbal, amenazas de abandono, chantaje...); nivel 3, tácticas que facilitan el acceso (aprovechar o propiciar el consumo de alcohol); y nivel 4, uso de la fuerza física.

Como puede verse, muchos de los instrumentos desarrollados para analizar la coerción sexual en las parejas presentan algún tipo de limitación: medir la coerción a lo largo de la vida sin distinguir el momento o la frecuencia, no diferenciar entre coerción y agresión sexual, etc. De ahí la necesidad de diseñar un instrumento que mida, específicamente, las tácticas de coerción sexual en parejas estables.

Volviendo a la relación entre coerción sexual y violencia, no sólo es de interés analizar la posible asociación entre ambas variables, sino también su conexión con el tipo de compromiso por el que se mantiene la pareja. Aunque la investigación sobre violencia ha señalado que la duración de una relación y el compromiso con la misma incrementan el riesgo de violencia (v. g. Sugarman y Hotaling, 1989), numerosos trabajos demuestran los beneficios del compromiso en las relaciones no violentas. Básicamente, el estudio del compromiso se ha abordado desde dos perspectivas (Fehr, 2001). Los trabajos centrados en analizar las variables que predicen el compromiso: nivel de satisfacción, calidad de las alternativas e inversión realizada (v. g. Levinger, 1965; Rusbult, 1983; Thibaut y Kelley, 1959); y los que tratan de identificar diferentes tipos de compromiso: personal (basado en la satisfacción), estructural o constraining (basado en los obstáculos para romper) y moral (asociado a valores religiosos o morales) (Adams y Jones, 1997; Johnson, 1973). Desde esta última perspectiva, González-Méndez y Hernández-Cabrera (2008) detectaron dos tipos de compromiso que predicen la victimización: restrictivo o negativo (que subraya los obstáculos para romper y aumenta el riesgo) y personal o positivo (que lo disminuye).

En definitiva, nos planteamos dos objetivos: 1) iniciar la construcción de una escala que mida coerción sexual en parejas estables; y 2) estudiar la relación entre las estrategias de coerción detectadas por la escala, el tipo de compromiso con la pareja y la violencia física y sexual. En este sentido, esperamos que las relaciones con mayor nivel de coerción sexual y compromiso negativo sean las que presenten más violencia física y sexual.
Método

\section{Participantes}

La muestra estaba integrada por 175 universitarios, 79 mujeres y 96 hombres de cinco titulaciones, con relaciones heterosexuales de cierta duración $(M=2.70$ años, $D T=2.39)$, y actividad sexual que incluía la penetración. Tenían edades entre los 18 y los 35 años $(M=22.6, D T=3.35)$. El 69.70\% tenía pareja (55 mujeres y 67 hombres) frente al $30.30 \%$ que no la tenía (24 mujeres y 29 hombres), pero respondió refiriéndose a una relación ya terminada. El $60.60 \%$ no convivía con su pareja (50 mujeres y 56 hombres) y el $39.40 \%$ sí (29 mujeres y 40 hombres). El $81.40 \%$ no tenía gastos compartidos y el $18.60 \%$ sí los tenía. Sus parejas tenían estudios universitarios en el $62.40 \%$ de los casos.

\section{Material y procedimiento}

Junto a diversas preguntas relacionadas con los participantes y sus parejas, el instrumento que elaboramos incluía varias medidas: a) una escala de coerción sexual para parejas que se puso a prueba en este estudio; b) una versión reducida de la CTS (Straus, 1979) para medir violencia física, que ya fue probada por González-Méndez y Hernández-Cabrera (2008); y c) una escala de compromiso con la relación, propuesta y verificada por esos mismos autores. Finalmente, se añadieron dos ítems, construidos para este estudio, que medían la agresión sexual sufrida por las mujeres. En concreto, se les preguntaba: 1) frecuencia con la que se ha visto forzada a mantener contacto sexual (sin penetración); y 2) frecuencia con la que se ha visto forzada a mantener contacto sexual (con penetración). En ambos casos, las puntuaciones de frecuencia iban de 0 (nunca) a 10 (siempre). No obstante, decidimos utilizar sólo la opción "con penetración" tras comprobar que la correlación entre ambas medidas era .99 .

Coerción Sexual. Para elaborar esta escala, comenzamos revisando las investigaciones previas y realizando entrevistas a universitarios de distintos sexos $(\mathrm{n}=10)$, que no participaron en el estudio definitivo. La revisión señaló la necesidad de diseñar un instrumento que diferenciara claramente entre coerción y agresión, y que se adaptara a las características de las relaciones estables. Para construir los ítems, tuvimos en cuenta las dimensiones halladas por otras investigaciones. No obstante, no incluimos el uso de alcohol y otras substancias psicoactivas por dos razones. Por un lado, en las entrevistas previas, se apuntó su escasa frecuencia en parejas estables. Por otra parte, algunos investigadores han sugerido que la coerción sexual que se produce en los encuentros informales o las citas puede ser diferente de la que se da en el contexto de las relaciones de pareja (Shackelford y Goetz, 2004), y precisamente en los incidentes de abuso sexual asociados con consumo de alcohol, es más frecuente que los agresores sean poco conocidos por la víctima (Abbey, Zawacki, Buck, Clinton y McAuslan, 2004; Ullman, 2003). 
La escala incluye 16 ítems idénticos para ambos sexos, de los cuales sólo 13 fueron considerados al evaluar la coerción. Los otros tres se añadieron para ofrecer alternativas no coercitivas ("siempre estoy dispuesto/a", "se insinúa con caricias, diciéndome cosas" y "lo comprende y no insiste"). En primer lugar, se pidió a todos los participantes que indicaran la frecuencia con la que sus parejas utilizan distintas tácticas para conseguir una relación sexual no deseada por ellos/as (coerción sufrida). En segundo lugar, se les preguntaba también a todos por la frecuencia con la que ellos/as mismos/as utilizan esas tácticas (coerción ejercida). En cada caso, la frecuencia se midió a través de escalas que iban de 0 (nunca) a 10 (siempre). A partir de una aplicación única, se hallaron las alphas de Cronbach: .80 para la escala de coerción sufrida y .77 para la de coerción ejercida.

Violencia fisica sufrida. Para medir la violencia física sufrida, se utilizó una versión reducida de la Conflict Tactics Scales (CTS; Straus, 1979), que ya ha sido probada anteriormente (v. g. González-Méndez y Hernández-Cabrera, 2008). La escala consta de 10 ítems, de los cuales seleccionamos dos para medir violencia física ("empujar al otro/a" y "pegar al otro/a") ( $\alpha=$ .84). Los participantes tenían que indicar la frecuencia con la que sus parejas manifiestan diversas conductas durante sus conflictos. El rango de puntuaciones iba de 0 (nunca) a 10 (siempre).

Compromiso con la relación. Para conocer el tipo de compromiso con la relación, utilizamos la escala propuesta por González-Méndez y Hernández-Cabrera (2008). Este instrumento presenta diversas razones para mantener una relación de pareja, y pide a los participantes que indiquen su grado de acuerdo con cada una de ellas, en una escala que va de 0 (totalmente en desacuerdo) a 10 (totalmente de acuerdo). A pesar de haber sido probada anteriormente, realizamos un análisis de componentes principales que confirmó la agrupación de los ítems en dos factores que explican el $74.06 \%$ de la varianza. Un tipo de Compromiso Positivo ("nos apoyamos" (.93) y "estamos bien juntos" (.90); que explica el $43.80 \%$ de la varianza), y un Compromiso Negativo ("mi pareja insiste para que sigamos juntos" (.89), "yo insisto para que sigamos juntos" (.86), "es difícil romper" (.81), y "tengo miedo a quedarme solo/a" (.65); que explica el $30.26 \%)$. El test de esfericidad de Bartlett resultó significativo $\left(\chi^{2}(15)=452.43 ; p<.001\right)$; el test KMO fue de .70. El alpha de Cronbach de las sub-escalas fue de .84 y .82 , respectivamente.

Tras pedir permiso al profesorado de distintos centros, se administró el cuestionario en sus aulas. Los participantes respondieron de forma voluntaria y anónima, y nadie rehusó colaborar, aunque no se ofrecieron créditos. Aquellos que no tenían pareja en ese momento, respondieron con relación a una pareja anterior.

$$
\text { Resultados }
$$

Análisis psicométrico de la escala de coerción sexual en parejas estables

Para analizar la estructura interna de la escala, hicimos dos análisis factoriales exploratorios (componentes principales), uno con la coerción sufrida y otro con la coerción ejercida. Previamente, comprobamos los supuestos necesarios para este tipo de análisis, y excluimos varios ítems que presentaban una asimetría y apuntamiento elevados, atribuible a su baja frecuencia entre los universitarios. Concretamente, eliminamos: "mi pareja amenaza con dejarme"; "amenaza con irse con otro/a"; "golpea objetos discutiendo por ese motivo"; "yo amenazo con dejar la relación"; "yo amenazo con irme con otra/o" y "уо golpeo objetos discutiendo por ese motivo".

Respecto a la coerción sufrida, el test de esfericidad de Bartlett resultó significativo $\left(\chi^{2}(45)=544.80 ; p<.001\right)$; el test KMO de adecuación de la muestra fue de .81 ; y los valores del MSA se situaron, en su totalidad, por encima de .70. Tras esta comprobación, se detecta una estructura factorial de tres componentes (mediante rotación ortogonal, Varimax), que explica el 63.36\% de la varianza total (ver tabla 1). El primer componente $(22.57 \%$ de la varianza explicada), Chantaje Emocional, está formado por 3 ítems que suponen retirada de afecto. El segundo componente (22.40\%), Culpabilización, agrupa 4 ítems relacionados con acusaciones y reproches. Finalmente, la Insistencia (18.39\%) integra 3 ítems que implican persistencia en la demanda sexual.

Con relación a la Coerción Ejercida, el test de esfericidad de Bartlett resultó igualmente significativo $\left(\chi^{2}(45)=558.22\right.$; $p<.001$ ); el test KMO fue de .78; y los valores MSA se situaron por encima de .70. Los análisis revelan una solución de 3 componentes (rotación ortogonal, Varimax) que explican el 65.28\% de la varianza total (ver tabla 1). Los ítems vuelven a agruparse igual que en el análisis anterior. No obstante, la Culpabilización explica ahora mayor porcentaje de varianza $(24.62 \%)$ que el Chantaje Emocional (21.20\%). El porcentaje de varianza explicado por la Insistencia es $19.46 \%$.

Tabla 1.

Análisis de componentes principales con los ítems de la escala de coerción sufrida.

\begin{tabular}{lccc}
\hline \multirow{2}{*}{ Ítems de la escala } & \multicolumn{3}{c}{ Componentes } \\
\cline { 2 - 4 } & $\begin{array}{c}\text { Chantaje } \\
\text { Emocional }\end{array}$ & Culpabilización & Insistencia \\
\hline Deja de ser cariñoso & .890 & & \\
Se pone serio/a & .786 & .347 & \\
Se pone triste & .705 & .849 & \\
\hline $\begin{array}{l}\text { Dice que con otro/as no } \\
\text { le ocurría }\end{array}$ & & .658 & \\
$\begin{array}{l}\text { Dice que quizás le soy } \\
\text { infiel }\end{array}$ & & .638 & .812 \\
$\begin{array}{l}\text { Discute por cualquier } \\
\text { motivo }\end{array}$ & .328 & .632 & .695 \\
$\begin{array}{l}\text { Dice que se tiene que ir } \\
\text { Sigue seduciéndome } \\
\text { con caricias }\end{array}$ & .341 & & .676 \\
$\begin{array}{l}\text { Justifica su necesidad } \\
\text { de sexo }\end{array}$ & & & $18.39 \%$ \\
$\begin{array}{l}\text { Regatea una felación/ } \\
\text { masturbación }\end{array}$ & & $22.40 \%$ & \\
\hline $\begin{array}{l}\text { 63.36\% varianza total } \\
\text { explicada }\end{array}$ & $22.57 \%$ & & \\
\hline
\end{tabular}


Análisis de componentes principales con los ítems de la escala de coerción ejercida.

\begin{tabular}{lccc}
\hline & \multicolumn{3}{c}{ Componentes } \\
\cline { 2 - 3 } \multicolumn{1}{c}{ Ítems de la escala } & Culpabilización & $\begin{array}{c}\text { Chantaje } \\
\text { Emocional }\end{array}$ & Insistencia \\
\hline $\begin{array}{l}\text { Dices que con otros/as } \\
\text { no te ocurría }\end{array}$ & .829 & & \\
$\begin{array}{l}\text { Dices que tienes que } \\
\text { irte }\end{array}$ & .738 & .350 & \\
$\begin{array}{l}\text { Dices que quizás te } \\
\text { sea infiel }\end{array}$ & .678 & & .405 \\
$\begin{array}{l}\text { Discutes por cualquier } \\
\text { motivo }\end{array}$ & .637 & & \\
\hline $\begin{array}{l}\text { Dejas de ser cariñoso/a } \\
\text { Te pones triste }\end{array}$ & & .826 & \\
Te pones serio/a & .421 & .734 & .794 \\
\hline $\begin{array}{l}\text { Justificas tu necesidad } \\
\text { de sexo }\end{array}$ & & & .788 \\
$\begin{array}{l}\text { Regateas una felación/ } \\
\text { masturbación }\end{array}$ & & & .653 \\
$\begin{array}{l}\text { Sigues seduciéndolo/a } \\
\text { con caricias }\end{array}$ & & & $19.46 \%$ \\
\hline $\begin{array}{l}\text { 65.28\% varianza total } \\
\text { explicada }\end{array}$ & $24.62 \%$ & $21.20 \%$ & \\
\hline
\end{tabular}

Nota: se incluyen, en gris, los pesos de algunos de los ítems en otros factores.

Análisis descriptivo de la coerción sexual: rangos y porcentajes

Tras hallar la media aritmética de cada factor de coerción con las frecuencias de los ítems incluidos en cada uno de ellos, establecimos tres rangos con los promedios de frecuencia. Un primer rango, que denominamos ausencia de coerción sexual, con los casos que puntúan 0 en las dos medidas de coerción (sufrida y ejercida). Un segundo rango, coerción de baja frecuencia, con los que puntúan por encima de cero y por debajo de 5, al menos, en una de las dos medidas de coerción. Por último, llamamos coerción de alta frecuencia al rango que agrupa los casos con puntuaciones superiores a 5, al menos, en una de las dos medidas de coerción. A partir de aquí, calculamos el porcentaje de parejas afectadas por cada tipo de coerción sexual.

Tabla 2. Porcentaje de parejas afectadas por cada tipo de coerción sexual, considerando coerción sufrida y ejercida conjuntamente.

\begin{tabular}{lccc}
\hline Tipos de coerción & Porcentaje total & $\begin{array}{c}\text { Porcentaje con } \\
\text { baja frecuencia }\end{array}$ & $\begin{array}{c}\text { Porcentaje con } \\
\text { alta frecuencia }\end{array}$ \\
\hline Insistencia & 88.20 & 63.60 & 24.60 \\
$\begin{array}{l}\text { Chantaje } \\
\text { Emocional }\end{array}$ & 73.80 & 49.60 & 24.20 \\
Culpabilización & 46.00 & 43.60 & 2.40 \\
\hline
\end{tabular}

La tabla 2 muestra que la forma de coerción más extendida es la Insistencia sexual (88.20\%), con baja frecuencia en un $63.60 \%$ de los casos, y alta frecuencia en un $24.60 \%$. Seguidamente, el Chantaje Emocional afecta al $73.80 \%$, (baja frecuencia en un $49.60 \%$ y alta en un $24.20 \%$ ). Finalmente, la forma de coerción menos extendida es la Culpabilización (46\%), que es además la menos frecuente, puesto que la proporción relativa de parejas con baja frecuencia (43.60\%) frente a las de alta frecuencia $(2.40 \%)$ es mayor que en las otras formas de coerción.

\section{Correlaciones}

Como evidencia de la validez externa de la escala (validez de criterio), hallamos las correlaciones entre las sub-escalas de coerción sexual, los dos factores de compromiso y la violencia sufrida. La tabla 3 muestra correlaciones significativas entre la violencia sufrida y las tres sub-escalas de coerción sufrida, pero no con las de Coerción Ejercida. El Compromiso Positivo correlaciona negativamente con todas las tácticas salvo con Insistencia; mientras que el Compromiso Negativo correlaciona con todas las de Coerción Sufrida y con Chantaje Ejercido.

Tabla 3. Correlaciones entre las tácticas de coerción sexual, los dos tipos de compromiso y la violencia física sufrida.

\begin{tabular}{lccccccccc}
\hline & 1 & 2 & 3 & 4 & 5 & 6 & 7 & 8 & 9 \\
\hline $\begin{array}{l}\text { 1. Chantaje } \\
\text { sufrido }\end{array}$ & 1.00 & .000 & .000 & $.320^{* *}$ & .031 & .009 & $-.206^{* *} .244^{* *}$ & $.251^{* *}$ \\
$\begin{array}{l}\text { 2. Culpa } \\
\text { sufrida }\end{array}$ & .000 & 1.00 & .000 & -.017 & $.507^{* *}$ & .042 & $-.198^{*}$ & $.189^{*}$ & $.258^{* *}$ \\
$\begin{array}{l}\text { 3. Insistencia } \\
\text { sufrida }\end{array}$ & .000 & .000 & 1.00 & -.027 & .071 & $.519^{* *}$ & -.072 & $.171^{*}$ & $.243^{* *}$ \\
$\begin{array}{l}\text { 4. Chantaje } \\
\text { ejercido }\end{array}$ & $.320^{* *}$ & -.017 & -.027 & 1.00 & .000 & .000 & $-.180^{*}$ & $.223^{* *}$ & .053 \\
$\begin{array}{l}\text { 5. Culpa } \\
\text { ejercida }\end{array}$ & .031 & $.507^{* *}$ & .071 & .000 & 1.00 & .000 & $-.415^{* *}$ & -.020 & .109 \\
$\begin{array}{l}\text { 6. Insistencia } \\
\text { ejercida }\end{array}$ & .009 & .042 & $.519^{* *}$ & .000 & .000 & 1.00 & -.009 & .001 & .127 \\
$\begin{array}{l}\text { 7. Compromiso } \\
\text { positivo }\end{array}$ & $-.206^{* *}$ & $-.198^{*}$ & -.072 & $-.180^{*}$ & $-.415^{* *}$ & -.009 & 1.00 & .000 & $-.222^{* *}$ \\
$\begin{array}{l}\text { 8. Compromiso } \\
\text { negativo }\end{array}$ & $.244^{* *}$ & $.189^{*}$ & $.171^{*}$ & $.223^{* *}$ & -.020 & .001 & .000 & 1.00 & $.299^{* *}$ \\
$\begin{array}{l}\text { 9. Violencia } \\
\text { sufrida }\end{array}$ & $.251^{* *}$ & $.258^{* *}$ & $.243^{* *}$ & .053 & .109 & .127 & $-.222^{* *} .299^{* *}$ & 1.00 \\
\hline
\end{tabular}

$* * \mathrm{p}<.01 * \mathrm{p}<.05$

Análisis discriminante

Para confirmar la asociación esperada entre violencia física, coerción sexual y tipo de compromiso realizamos un análisis discriminante paso a paso. Partimos de dos grupos con máxima diferenciación respecto a la violencia física sufrida. El primero estaba formado por las personas que puntúan 0 en los dos ítems de violencia física seleccionados (empujar y pegar); y el segundo estaba integrado por las que puntúan distinto de cero en alguno de esos ítems. Como variables discriminantes utilizamos los tres factores de coerción sufrida, los tres de coerción ejercida, y los dos de compromiso.

La correlación canónica resultante fue .42. Tras comprobar la igualdad de sus matrices de covarianzas mediante la prueba de Box, se confirman las diferencias significativas entre los dos grupos $\left(\Lambda=.83 ; \chi^{2}(3, N=159)=30.04 ; p<.001\right)$. La función que más discrimina entre ambos está formada por el Compromiso Negativo, el Compromiso Positivo y la Insistencia sufrida (ver tabla 4). Esta función permite clasificar correctamente al $75.20 \%$ de los casos. Concretamente, el $76.10 \%$ del grupo sin violencia y el $68.40 \%$ del grupo con violencia fueron clasificados correctamente. 
Tabla 4. Datos de las variables incluidas en la función que discrimina entre el grupo que ha sufrido violencia física y el grupo que no la ha sufrido.

\begin{tabular}{lccccc}
\hline $\begin{array}{l}\text { Variables en } \\
\text { la función } \\
\text { discrimi- } \\
\text { nante }\end{array}$ & $\begin{array}{c}\text { Coeficientes } \\
\text { tipificados }\end{array}$ & \multicolumn{2}{c}{$\begin{array}{c}\text { Grupo que sufre } \\
\text { violencia }\end{array}$} & \multicolumn{2}{c}{$\begin{array}{c}\text { Grupo que no sufre } \\
\text { violencia }\end{array}$} \\
\hline $\begin{array}{l}\text { Compromiso } \\
\text { Negativo }\end{array}$ & 0.74 & 4.29 & 2.40 & 1.82 & 2.19 \\
\hline $\begin{array}{l}\text { Compromiso } \\
\text { Positivo }\end{array}$ & -0.54 & 7.05 & 1.80 & 8.45 & 1.72 \\
\hline $\begin{array}{l}\text { Insistencia } \\
\text { sufrida }\end{array}$ & 0.38 & 8.61 & 6.33 & 7.10 & 5.24 \\
\hline
\end{tabular}

Valor de los centroides: grupo que sufre violencia $=1.24$; grupo que no sufre violencia $=-0.17$

\section{Violencia sexual}

Los casos donde se detecta sexo forzado con penetración son diez, lo que supone el $12,60 \%$ de las mujeres participantes. De ellas, la mitad también sufre violencia física. De hecho, la correlación entre ambas formas de violencia es significativa ( $r$ $=.60 ; p<.001)$. Asimismo, se observa que todas sufren Chantaje, todas menos una sufren Insistencia, y sólo la mitad sufre Culpabilización. Finalmente, detectamos correlaciones moderadas, pero significativas, entre la Violencia Sexual y dos de las tácticas de coerción sexual: Culpabilización $(r=.35 ; p<.001)$ y Chantaje $(r=.33 ; p<.001)$; así como entre la Violencia Sexual y el Compromiso Negativo $(r=.30 ; p<.001)$.

\section{Discusión}

Con relación al primer objetivo, el análisis psicométrico de la escala de coerción sexual ofrece evidencia preliminar de su coherencia interna y su fiabilidad. Asimismo, las correlaciones entre las tácticas de coerción y las otras variables analizadas aportan indicios de validez de criterio.

El análisis exploratorio realizado con las tácticas de coerción sexual revela una estructura de tres componentes, que se repite al analizar la coerción sufrida y la ejercida. Estos factores son el Chantaje Emocional, que supone la retirada de afecto (deja de ser cariñoso/a, se pone triste o serio/a); la Culpabilización, que señala o sugiere algún tipo de problema en la pareja ("con otras parejas no le ocurría", "puede que le estés siendo infiel"), pero que también incluye reacciones como discutir o marcharse; y la Insistencia, consistente en mantener la presión a pesar de la negativa expresa de la pareja ("sigue seduciéndome con palabras o caricias", "justifica su necesidad de sexo" y "regatea una felación/masturbación”). Los dos primeros factores se asemejan a la persuasión verbal negativa definida por Livingston et al. (2004) y a la manipulación emocional descrita por Stuckman-Johnson et al. (2003). Sin embargo, nuestros resultados distinguen entre ambas, mostrando además que el chantaje emocional afecta a mayor número de parejas y es más frecuente que la culpabilización. Por otro lado, la insistencia resultó ser la estrategia más extendida, a pesar de que no siempre aparece claramente delimitada en otras investigaciones.

Con relación al segundo objetivo, los resultados confirman la asociación esperada entre tipo de compromiso, coerción sexual y ambas formas de violencia.

En primer lugar, quienes mantienen su relación debido a las dificultades para romper tienen mayor probabilidad de sufrir tanto coerción sexual como violencia física. En cambio, quienes aseguran estar bien juntos y apoyarse presentan menos riesgo. Los datos que relacionan compromiso y violencia física concuerdan con los resultados de González-Méndez y Hernández-Cabrera (2008), y señalan que el riesgo de victimización aumenta cuando el compromiso con la relación es negativo, pero disminuye cuando es positivo. No obstante, la relación detectada entre compromiso y coerción sexual no ha sido descrita anteriormente. En conjunto, estos datos señalan la importancia del tipo de compromiso para comprender y predecir la dinámica de las relaciones, indicando que el compromiso negativo incrementa el riesgo de coerción y de violencia.

Por otro lado, los resultados también confirman la asociación entre las tres sub-escalas de coerción sufrida y la violencia física, tal como se ve a través de las correlaciones. No obstante, en el análisis discriminante la insistencia sufrida adquiere más relevancia de lo esperado, actuando como variable supresora frente a las otras dos formas de coerción. La insistencia aparece además como un factor de riesgo específico de la violencia física, ya que no correlaciona con la violencia sexual. Algunos trabajos sugieren que las estrategias de coerción más comunes son las que implican menor gravedad (Livingston et al., 2004; Struckman-Johnson et al., 2003). No obstante, nuestros datos no apoyan esta interpretación. Aunque la prevalencia relativa detectada para las diferentes tácticas de coerción sexual es similar a la hallada en otros estudios, y la insistencia aparece como la estrategia más común, seguida de la manipulación emocional (v. g. Struckman-Johnson et al., 2003), esta estrategia también emerge como factor de riesgo respecto a la violencia física.

Finalmente, los resultados indican una correlación elevada entre violencia física y sexual, pero la presencia de sexo forzado con penetración no pudo utilizarse en el análisis discriminante por haber sido medido únicamente en las mujeres. Con todo, el análisis de casos sugiere que la violencia sexual puede ser mejor predictor de la violencia física, que al revés. Así, mientras la mitad de las mujeres que sufren violencia sexual también sufren violencia física, sólo la cuarta parte de las sufren violencia física informan de penetración forzada. Estos datos concuerdan con los de otros estudios donde se ha detectado que la violencia sexual suele ir asociada a la violencia física, pero no necesariamente al revés (v.g. Harner, 2002).

La mayor parte de los estudios realizados en España han analizado la coerción y la agresión sexual en relaciones esporádicas (v. g. Fuertes, Ramos, Orden, Campo y Lázaro, 2005; Sipsma, Carrobles, Montorio y Everaerd, 2000). Así, por ejemplo, Sipsma et al. (2000) encontraron que el $33.20 \%$ de las 
universitarias admitía haber sido víctima de alguna forma de coerción sexual por parte de algún conocido. Sin embargo, el presente estudio indica que estas prácticas están aún más extendidas entre las parejas. Tal como plantean Medina-Ariza, y Barberet (2003), las mujeres que sufren abuso sexual por parte de sus parejas tienen menos probabilidad de verse a sí mismas como víctimas de violencia. Aunque en los últimos años, ha crecido el rechazo social hacia el maltrato físico, la coerción sexual sigue siendo tolerada. Sin embargo, su elevada prevalencia no reduce el riesgo que conlleva para las relaciones. En este sentido, es necesario que los programas educativos de prevención de la violencia aborden la identificación de estrategias de coerción sexual y el cambio de actitudes hacia tales prácticas. Asimismo, conviene resaltar el riesgo asociado al compromiso negativo.

Este estudio tiene carácter exploratorio y, como tal, presenta limitaciones importantes. Los datos psicométricos y la evidencia de validez de criterio de la escala de coerción sexual son preliminares. Por lo tanto, es imprescindible disponer de una muestra mucho más amplia y heterogénea, que permita hacer una validación cruzada de los resultados. Asimismo, resulta necesario obtener evidencias de validez convergente y discriminante. Convendría también establecer el nivel de gravedad de las diferentes estrategias, no sólo por su relación con la violencia, sino también por sus efectos físicos y psicológicos. En este sentido, es necesario analizar cómo afectan tales estrategias a hombres y mujeres.

\section{Referencias}

Abbey, A., Zawacki, T., Buck, P.O., Clinton, A.M. y McAuslan, P. (2004). Sexual assault and alcohol consumption: What do we know about their relationship and what types of research are still needed? Aggression and Violent Behavior, 9, 271-303.

Adams, J.M. y Jones, W.H. (1997). The conceptualization of marital commitment: An integrative analysis. Journal of Personality and Social Psychology, 72, 1177-1196.

Adams-Curtis, L.E. y Forbes, G.B. (2004). College women's experiences of sexual coercion: A review of cultural, perpetrator, victim, and situational variables. Trauma, Violence \& Abuse, 5, 91-122.

DeGue, S. y DiLillo, D. (2005). "You would if you loved me": Toward an improved conceptual and etiological understanding of nonphysical male sexual coercion. Aggression and Violent Behavior, 10, 513-532.

Fehr, B. (2001). The status of theory and research on love and commitment. In G. J. O. Fletcher \& M. S. Clark (Eds.), Blackwell handbook of social psychology: Interpersonal processes (pp. 331-356). London: Basil Blackwell.

Fernández-Fuertes, A.A., Fuertes, A. y Pulido, R.F. (2006). Evaluación de la violencia en las relaciones de pareja de los adolescentes. Validación del Conflict in Adolescent Dating
Relationships Inventory (CADRI) - versión española. International Journal of Clinical and Health Psychology, 6, 339-358.

Fuertes, A., Ramos, M., de la Orden, V., del Campo, A. y Lázaro, S. (2005). The involvement in sexual coercive behaviors of Spanish college men. Prevalence and risk factors. Journal of Interpersonal Violence, 20, 872-891.

González, R. y Santana, J. D. (2001). Violencia en parejas jóvenes. Psicothema, 13, 127-131.

González-Méndez, R. y Hernández-Cabrera, J.A. (2008). Play context, commitment, and dating violence. A structural equation model. Journal of Interpersonal Violence. DOI: $10.1177 / 0886260508323666$.

Harner, M.S. (2002). A multivariate analysis of risks markers for dating violence victimization. Journal of Interpersonal Violence, 17, 1179-1197.

Jewkes, R. (2002). Intimate partner violence: causes and prevention. The Lancet, 359, 1423-1429.

Johnson, M. P. (1973). Commitment: A conceptual structure and empirical application. Sociological Quarterly, 14, 395406.

Koss, M.P. y Oros, C.J. (1982). Sexual Experiences Survey: A research Instrument Investigating Sexual Aggression and Victimization. Journal of Consulting and Clinical Psychology, 50, 455-457.

Krug, E., Dahlberg, L. y Mercy, J. (2002). World report on violence and health. Washington: World Health Organization.

Levinger, G. (1965). Marital cohesiveness and dissolution: An integrative review. Journal of Marriage and Family, 27, 19-28.

Lewis, S.F. y Fremouw, W. (2001). Dating violence: A critical review of the literature. Clinical Psychology Review, 21, 105-127.

Livingston, J.A., Buddie, A.M., Testa, M. y VanZile-Tamsen, C. (2004). The role of sexual precedence in verbal sexual coercion. Psychology of Women Quarterly, 28, 287-297.

Medina-Ariza, J. y Barberet, R. (2003). Intimate Partner Violence in Spain Findings From a National Survey. Violence Against Women, 9, 302-322.

Muehlenhard, L.C. y Peterson, Z. D. (2005). Conceptualizing Sexual Violence: Socially Acceptable Coercion and Other Controversies. En A. G. Miller (Ed.), The Social Psychology of Good and Evil (pp. 240-268). New York: Guildford Press.

Muñoz-Rivas, M.J., Graña, J.L., O’Leary, K.D., y González, P. (2007). Physical and psychological aggression in dating relationships in Spanish university students. Psicothema, 19, 102-107.

Rusbult, C. E. (1983). A longitudinal test of the investment model: The development (and deterioration) of satisfaction and commitment in heterosexual involvements. Journal of Personality and Social Psychology, 45, 101-117. 
Serapio, A. (2006). Realidad psicosocial: La adolescencia actual y su temprano comienzo. Revista de Estudios de Juventud, 73, 11-23.

Shackelford, T. K., y Goetz, A. T. (2004). Men's sexual coercion in intimate relationships: Development and initial validation of the Sexual Coercion in Intimate Relationships Scale. Violence and Victims, 19, 541-556.

Sipsma, E., Carrobles, J.A., Montorio, I. y Everaerd, W. (2000). Sexual aggression against women by men acquaintances: Attitudes and experiences among spanish university students. The Spanish journal of Psychology, 3, 14-27.

Straus, M.A. (1979). Measuring intrafamily conflict and violence: The conflict tactics scale. Journal of Marriage and Family, 41, 75-88.

Straus, M.A., Hamby, S.L., Boney-McCoy, S., y Sugarman, D.B. (1996). The Revised Conflict Tactics Scales (CTS2): Development and Preliminary Psychometric Data. Journal of Family Issues, 17, 283-316.

Struckman-Johnson, C., Strucman-Johnson, D. y Anderson, P.B. (2003). Tactics of sexual coercion: When men and women won't take no for an answer. The Journal of Sex Research, 40, 76-86.

Sugarman, D. B. y Hotaling, G.T. (1989). Dating violence: prevalence, context, and risk markers. En M. A. PirogGood y J.E. Stets (eds.), Violence in dating relationship: Emerging social issues (pp. 3-31). Wesport, CT: Praeger.
Testa, M. y Dermen, K. H. (1999). The differential correlates of sexual coercion and rape. Journal of Interpersonal Violence, 14, 548-561.

Testa, M., VanZile-Tamsen, C. y Livingston, A. (2007). Prospective prediction of women's sexual victimization by intimate and Nonintimate male perpetrators. Journal of Consulting and Clinical Psychology, 75, 52-60.

Thibaut, J.W., y Kelley, H.H. (1959). The social psychology of groups. New York: John Wiley.

Ullman, S. E. (2003). A critical review of field studies on the link of alcohol and adult sexual assault in women. Aggression and Violent Behavior, 8, 471-486.

Vézina, J. y Hébert, M. (2007). Risk factors for victimization in romantic relationships of young women. A Review of Empirical Studies and Implications for Prevention. Trauma, Violence \& Abuse, 8, 33-66. DOI: $10.1177 / 1524838006297029$.

Fecha de recepción: 19 de mayo de 2009 Fecha de aceptación 7 de agosto de 2009 